University of Nebraska - Lincoln

DigitalCommons@University of Nebraska - Lincoln

CSE Conference and Workshop Papers

Computer Science and Engineering, Department of

2012

\title{
Empirical Analysis of the Hidden Terminal Problem in Wireless Underground Sensor Networks
}

Xin Dong

University of Nebraska-Lincoln, xdong@cse.unl.edu

Mehmet C. Vuran

University of Nebraska-Lincoln, mcvuran@cse.unl.edu

Follow this and additional works at: http://digitalcommons.unl.edu/cseconfwork

Dong, Xin and Vuran, Mehmet C., "Empirical Analysis of the Hidden Terminal Problem in Wireless Underground Sensor Networks" (2012). CSE Conference and Workshop Papers. 281.

http://digitalcommons.unl.edu/cseconfwork/281

This Article is brought to you for free and open access by the Computer Science and Engineering, Department of at DigitalCommons@University of Nebraska - Lincoln. It has been accepted for inclusion in CSE Conference and Workshop Papers by an authorized administrator of

DigitalCommons@University of Nebraska - Lincoln. 


\title{
Empirical Analysis of the Hidden Terminal Problem in Wireless Underground Sensor Networks
}

\author{
Xin Dong, Mehmet C. Vuran \\ Cyber-Physical Networking Laboratory \\ Department of Computer Science \& Engineering \\ University of Nebraska-Lincoln, Lincoln, NE 68588 \\ Email: \{xdong, mcvuran\}@cse.unl.edu
}

\begin{abstract}
Wireless Underground Sensor Networks (WUSNs) can be widely adopted to monitor the environment in agricultural, security and ecological applications. In most applications, besides underground sensor motes, aboveground motes are deployed to gather information. However, due to the different communication ranges of the channels, hidden terminal problem occurs when multiple underground motes send data to the aboveground mote. In this paper, experiments are conducted to reveal this problem in WUSNs empirically. In addition, an RTS/CTS scheme is implemented to eliminate this problem and it is shown that the communication success can be increased from $32 \%$ to $76 \%$. More importantly, despite its improvements in collisions, RTS/CTS schemes alone cannot guarantee successful multi-access operation due to the high variance in channel quality in WUSNs, which motivates further research in this area.
\end{abstract}

\section{INTRODUCTION}

Wireless Underground Sensor Networks (WUSNs) are a natural extension of Wireless Sensor Networks (WSNs) to the underground environment, where underground nodes are deployed to monitor the properties of the environment, such as soil moisture, salinity in agriculture applications, vibrations in border patrol applications and chemical levels in ecological applications [1]. In WSUN applications, besides underground nodes, aboveground nodes are deployed to gather information from the underground nodes. For these applications, three communication links exist: the underground to underground (UG2UG) link, the underground to aboveground (UG2AG) link, and the aboveground to underground (AG2UG) link [7], [8]. Due to the conductivity of the soil, in addition to the spreading loss, electromagnetic waves also suffer from additional attenuation in soil. Thus, among the three links, the communication ranges vary dramatically. This is illustrated in Fig. 1, where the different communication distances and the transitional regions of the underground-to-aboveground communications are shown. In our experiments, the communication ranges of the UG2AG and AG2UG links are found to be 7 to 10 times longer than the communication range of the UG2UG link.

Due to the disparity of the links, in WUSNs, underground nodes face the inevitable hidden terminal problem. When multiple underground nodes try to send packets to an aboveground node simultaneously, they cannot sense the existence of each other through carrier sensing. Hence packets collide

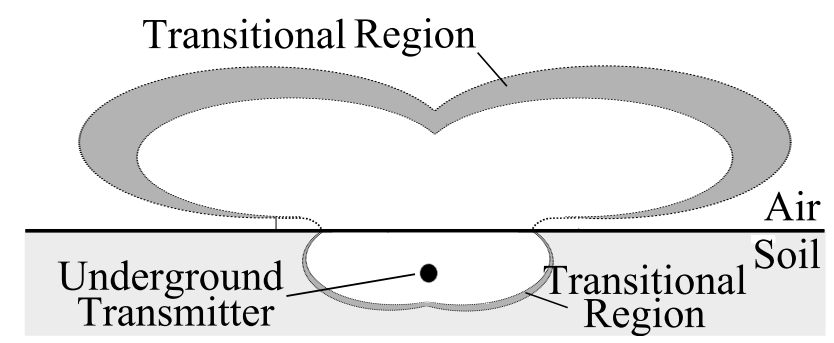

Fig. 1: The different communication ranges in underground-to-above ground communications

at the aboveground node. Even worse, in WUSN applications, aboveground nodes are sparsely deployed. Therefore, one aboveground node will gather information from tens to hundreds of underground nodes, which increases the probability of collision at the aboveground node.

In existing contention-based WSN MAC protocols, the RTS/CTS mechanism is employed to eliminate the hidden terminal problem [5]. Before a sender sends a data packet, it sends out a Request To Send (RTS) packet. Its neighbors that receive this packet refrain from sending data. The specified receiver, receiving the RTS packet, sends a Clear To Send (CTS) packet to the sender. This CTS packet stops its neighbors from sending data. In WUSN applications, by adopting this mechanism, the CTS sent by the aboveground nodes will notify the other underground nodes that the channel is busy. Thus, the collision at the aboveground node will be alleviated.

However, besides possible collisions, communications in WUSNs also suffer from high packet loss rate. This is mainly due to the high variance in channel quality in the air portion of the underground-to-aboveground channel [7]. In our experiments, it is shown that the packet loss rate can be as high as $30 \%$. Thus, in the MAC layer, the RTS or CTS packets may be lost. This causes the nodes to retransmit RTS packets and further increases the probability of collision.

In this paper, field experiments are conducted to evaluate the hidden terminal problem in WUSNs. An RTS/CTS scheme is implemented to evaluate the performance of this mechanism 
in WUSNs. The rest of the paper is organized as follows: In Section II, the related work is summarized. In Section III, test bed results are provided to evaluate the hidden terminal problem in WUSNs and in Section IV, the performance of RTS/CTS scheme in WUSNs is analyzed. The paper is concluded in Section V.

\section{RELATED WORK}

The concept and challenges of WUSNs have been introduced in [1]. In [3], [12], we develop a theoretical channel model for underground-to-underground (UG2UG) links. In [9], empirical UG2UG evaluations are realized confirming our previous theoretical findings. The communication links between underground and aboveground nodes are investigated empirically in [7], [8], [11]. The connectivity issues between underground and aboveground nodes are investigated in [10].

Despite the recent body of work, to the best of our knowledge, the MAC layer issues have not been investigated in the context of WUSNs. Conversely, in traditional WSNs, several MAC protocols have been developed [2]. In [14], the communication range of WSN nodes is investigated and the concept of transitional region is formalized. Based on empirical measurements, transitional region of a node is defined as the range at which communication errors are high enough to cause disruptions but not disconnection. A detailed investigation of the radio irregularities in WSNs is found in [13]. Based on these studies, in this work, contention aspects in the underground environment are investigated.

\section{HIDDEN TERMINAL EXPERIMENTS}

In this section, experiments are conducted to reveal the hidden terminal problem in WUSNs. B-MAC [6], which has been widely accepted in WSN applications and is implemented on Mica2, is employed. In addition to traditional CSMA/CAbased operation, B-MAC employs a Clear Channel Assessment (CCA) procedure, through which channel contention is detected based on periodic samples of the signal strength on the wireless channel. This detection occurs without the need for decoding the received data. Using the CCA overhearing mechanism, it is possible to better evaluate the limits of the different radio ranges of a node: communication, interference, and carrier sensing ranges [13], [14].

However, RTS/CTS scheme is not implemented in B-MAC. Thus, when hidden terminal occurs, B-MAC cannot prevent collisions since CCA is not capable of sensing the activity of the neighbor underground nodes.

\section{A. Experiment Setup}

Mica2 motes are employed for our experiments, and the frequency of the communication is $433 \mathrm{MHz}$. For the aboveground mote, a dipole antenna that is $90 \mathrm{~mm}$ long is used for the aboveground mote (master node). The antenna is placed vertically so the radiation pattern is a circle at the horizontal plane. For the underground motes (slave nodes), wideband planar antenna [4] is used. The antenna is placed horizontally to maximize the power transmitted through soil.

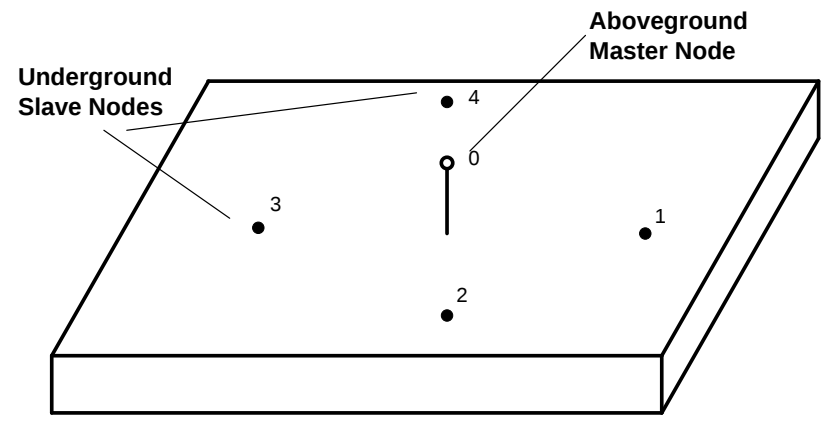

Fig. 2: The topology of the experiment

\begin{tabular}{|c||c|c|}
\hline Field & source & time \\
\hline Size (byte) & 2 & 4 \\
\hline
\end{tabular}

TABLE I: The structure of the beacon packet.

\begin{tabular}{|c||c|c|c|}
\hline Field & source & time & data \\
\hline Size (byte) & 2 & 4 & 14 \\
\hline
\end{tabular}

TABLE II: The structure of the data packet.

The deployment of our experiment is shown in Fig. 2, where the master node is placed in the middle of the field and is elevated to $1 \mathrm{~m}$ height. The slave nodes are buried at $10 \mathrm{~cm}$ underground and placed around the master node. The locations of the slave nodes are adjusted so that they cannot sense the existence of their neighbors but they can communicate with the master node. Horizontally, the slave nodes are $10 \mathrm{~m}-20 \mathrm{~m}$ away from the master node. In the experiment, we change the number of active slave nodes to analyze the collisions caused by the hidden terminal problem.

In the experiment, the master node sends out a beacon packet every $10 \mathrm{~s}$. After receiving the beacon, the slave nodes wait $0.1 \mathrm{~s}$ and then send the data packet to the master node. In each node, the sent packets and received packets are recorded. Note since the slave nodes do not have random back-off time, the case shown in this experiment is the worst case scenario.

The structures of the beacon packet and the data packet are shown in Table I-II, respectively.

\section{B. Experiment Results}

In this section, the packet loss caused by collisions is analyzed. In Fig. 3, the results of this experiment are shown in solid lines (CSMA/CA), including the average percentages of beacon packets received at the slave nodes, the data packets sent by the slave nodes and the DATA packets received by the master node are shown for different number of slave nodes. The highest percentage and the lowest percentage for each case are also shown. The percentages are normalized according to the number of beacon packets sent by the master node. We let the master node run about $30 \mathrm{~min}$. However, for the case of the 4 slave nodes, since the packet receiving rate is low at the master node, the experiment is run longer (about 1.5 hours). The number of beacons sent by the master node in each experiment is listed in Table III.

It is shown that the slave nodes have a high receiving rate 


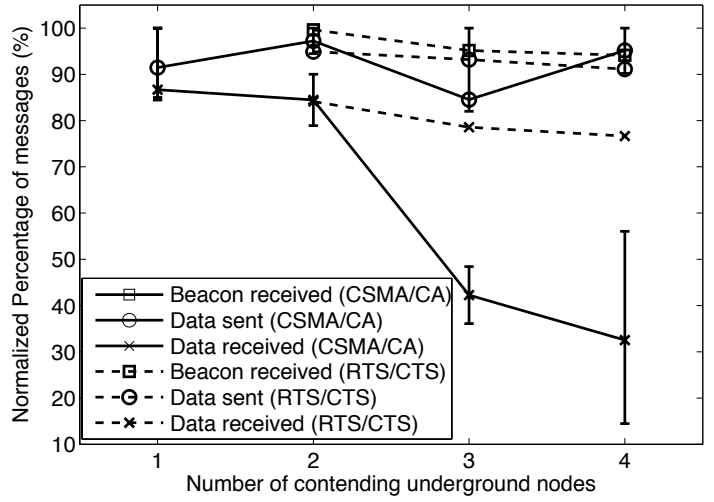

Fig. 3: Beacon received at the slave nodes, data sent by the slave nodes and data received at the master node.

\begin{tabular}{|c||c|c|c|c|}
\hline number of slave nodes & 1 & 2 & 3 & 4 \\
\hline number of beacons & 318 & 371 & 349 & 1024 \\
\hline
\end{tabular}

TABLE III: The number of beacons sent in each experiment.

of beacon packets. Except when the number of slave nodes is 3 , in which the success rate is $85 \%$, in other cases, the slave nodes received more than $90 \%$ of the beacon packets. This is due to the fact that when the master node is sending beacon, the channel is surely unoccupied. In other words, there is no interference. The cause for the drop in the success rate of three slave nodes is found to be due to the radio irregularity and the dynamic changes in the wireless channel.

Since the slave nodes send a data immediately after receiving a beacon, the number of data packets sent by each slave nodes is the same as the number of received beacon's. However, the master node does not receive all the data packets. First of all, due to radio irregularity and other factors, the packet loss is non-zero even if there is no interference from other nodes. This is shown in Fig. 3 when the number of the slave nodes is 1 , in which case the master node receives $86 \%$ of the packets on the average. The data packet reception rate decreases when the number of slave nodes increases due to collisions at the master node. The data packet reception rate is $43 \%$ even there are only three slave nodes and it decreases to only $32 \%$ when the number of slave nodes is 4. This collision problem is caused by the hidden terminal problem since the CCA scheme in B-MAC cannot sense the activity of the neighbor slave nodes. Thus, for a slave node, all of its peers are hidden terminals to it. Therefore, in WUSNs when underground-to-aboveground communication is considered, schemes to prevent hidden terminals needs to be implemented.

\section{RTS/CTS IN WUSNS}

In this section, we analyze the performance of the RTS/CTS scheme on the communication in hybrid WUSNs where underground-to-aboveground communication is employed.

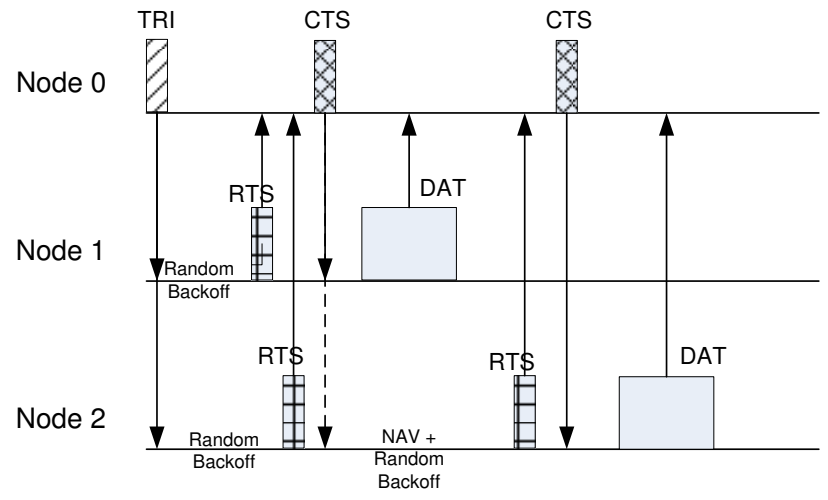

Fig. 4: The process of the RTS/CTS scheme implemented.

\begin{tabular}{|c||c|c|}
\hline Field & source & destination \\
\hline Size (byte) & 2 & 2 \\
\hline
\end{tabular}

TABLE IV: The structure of the RTS and CTS packets.

\begin{tabular}{|c||c|c|c|}
\hline number of slave nodes & 2 & 3 & 4 \\
\hline number of beacons & 409 & 412 & 421 \\
\hline
\end{tabular}

TABLE V: The number of beacons sent in each experiment.

\section{A. Implementation of RTS/CTS Scheme}

The topology of the network is the same as shown in Fig. 2. However, in addition to CCA, an RTS/CTS scheme is implemented. Note that the communication chip used in Mica2 (CC1000) does not support hardware RTS/CTS. Thus, this function is implemented at the application layer. The process of the scheme is shown in Fig. 4.

In Fig. 4, in addition to beacon and data packets, An RTS and a CTS packet are introduced. Both RTS and CTS packets are broadcast, however, there is a destination field in the message that the expected receiver is indicated. Both the RTS and CTS packets have the same structure, which is shown in Table IV

When the slave nodes receive a beacon packet, a random back-off is conducted, after which the slave nodes send out RTS's. The master node replies only to the first RTS and ignores the following ones. When a slave node overhears a CTS for its neighbors, it knows that it loses the competition and sleeps for network allocation vector (NAV) time plus another random back-off. The winning slave node which receives the CTS sends the data packet.

On Mica2, the time needed for an application layer communication is about $25 \mathrm{~ms}$. Therefore, in our experiments, the NAV time is set to $50 \mathrm{~ms}$ and the random back-off time is a random number between $0-50 \mathrm{~ms}$.

\section{B. Experiment Results}

The performance of the RTS/CTS scheme is analyzed in this section. The number of beacon packets sent in each experiment is shown in Table V. In Fig. 3, the results of this expriment are shown in dash lines, including the success rates of beacon packets received at the slave nodes, data packets sent by the slave nodes and DATA packets received at the master node 


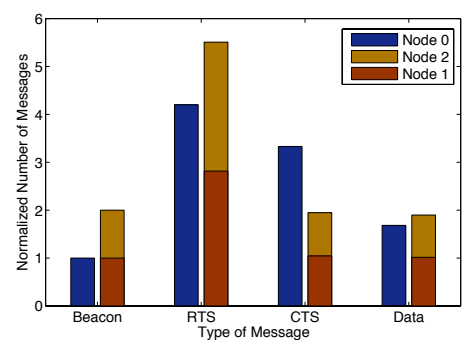

(a) 2 Slave Nodes

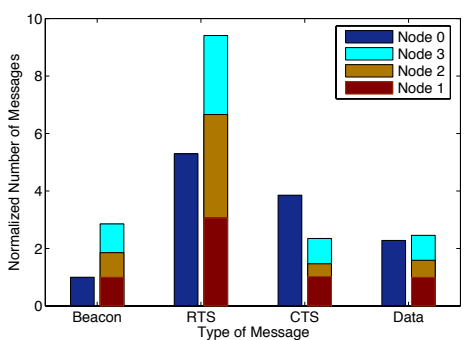

(b) 3 Slave Nodes

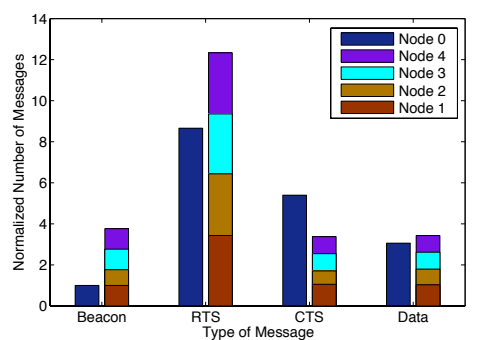

(c) 4 Slave Nodes

Fig. 5: beacon received at the slave nodes, data sent by the slave nodes and data received at the master node.

are shown. Compared with the results of CSMA/CA, it can be observed that when the number of slave nodes is small (2 slave nodes), the performance of the RTS/CTS scheme is worse than MAC without RTS/CTS. This is caused by high packet loss rate in WUSN communications. When RTS/CTS is adopted, to send a data, multiple small packets are sent to reserve the channel. However, if one of the packets is lost, the whole process will start again and hence the probability of failure is higher. For larger number of slave nodes, the benefit of the RTS/CTS scheme is shown. When the number of slave nodes is 3, the data receiving rate is $78.57 \%$, a 35 percentage increase compared to MAC without RTS/CTS. In addition, when the number of slave nodes is 4 , the data receiving rate is $76.64 \%$, a 44 percentage increase. Moreover, the data receiving rate does not drop dramatically as shown in the CSMA/CA case when increasing the number of slave nodes.

However, as shown in Fig. 3, the data receiving rate does not reach $100 \%$ even when RTS/CTS scheme is implemented. For all the beacon packets sent, the master node receives 76 $78 \%$ of the data packets. This is caused by the high packet loss rate in WUSNs. As shown in Section III, even when there is only one slave node, the master node receives $86 \%$ of the data packets.

The high packet loss rate also causes several RTS and CTS packets to be sent in the network. This is depicted in Fig. 5, where the number of each type of message sent/received from each node is shown. The number is normalized according to the number of beacon packets sent by the master node. As shown in the figures, to send a data packet, on average 2.9 RTS packets are sent and 1.75 CTS packets are sent when the number of slave nodes is 2 . The values are 3.83 and 1.57 , respectively, when the number of slave nodes is 3 and 3.59 and 1.57 , respectively, when the number of slave nodes is 4 . This certainly increases the energy consumption of the network. Moreover, as revealed, the cause of the high number of RTS packets is that the neighbor slave nodes can only be silenced by the CTS packets, and thus the RTS packets collide at the master node. One can increase the random back-off time to alleviate the problem at the master node. However, doing so will limit the number of slave nodes a master node can communicate within a given time.

The high packet loss of the RTS/CTS packets is also shown

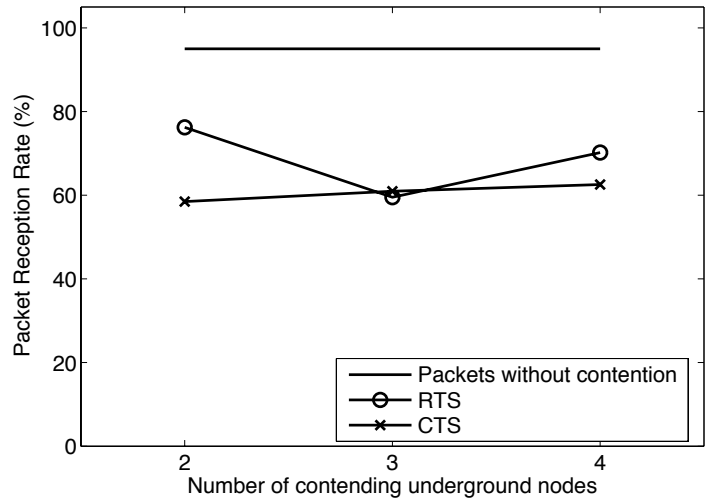

Fig. 6: The success rate of the RTS and the CTS packets.

in Fig. 6, where the packet reception rates of the RTS and CTS packets are depicted over the number of slave nodes. The packet reception rate without contention, as calculated in the 1 slave node case, is also shown. Due to the fact that during channel reserving process there is no collision prevention, the packet loss rate for RTS/CTS packets is much higher than other packets. Only $60-79 \%$ of packets are successfully received at the corresponding receivers. The low success rate of RTS/CTS packets is the main reason that this scheme does not perform as expected in WUSN communications.

Due to RTS/CTS contention, the delay of the DATA packets also increases. This is shown in Fig. 7, where the delay is calculated as the time difference between the master node sends a beacon and it receives the data. It is revealed that without RTS/CTS scheme, the delay does not increase dramatically when the number of underground nodes increases. When there is no underground node, the average delay is $99.49 \mathrm{~ms}$, and when there are 4 nodes, the average delay is $127.86 \mathrm{~ms}$. However, when RTS/CTS is adopted, the delay increases compared with the scenario without RTS/CTS. Even though the minimum delay is comparable to the scenario without RTS/CTS, the maximum delay increases 5.07-6.56 times. Moreover, the delay also increases with the increase of the number of underground nodes. When there are two contending nodes, the average delay is $263.95 \mathrm{~ms}$, and it 


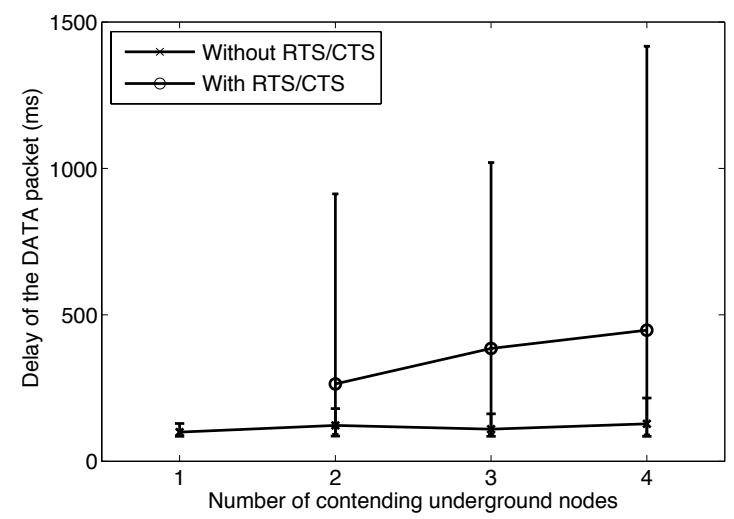

Fig. 7: The delay of the DATA packets in the two schemes.

increases to $385.34 \mathrm{~ms}$ when there are three contending nodes. For the case of the four contending nodes, the average delay is $447.74 \mathrm{~ms}$, an $69.63 \%$ increase compared with two nodes.

\section{CONCLUSiOns}

In this paper, empirical evaluations are conducted to investigate the hidden terminal problem in WUSNs. It is shown through experiments that due to the fact that the underground nodes cannot sense the existence of their underground neighbors, the CCA mechanism used in traditional WSNs is not sufficient to prevent collision at the aboveground nodes. The impacts of the RTS/CTS scheme is also evaluated through experiments. It is shown that even though this scheme can improve data packet reception rate at the aboveground node, the high packet loss rate in WUSNs, especially during RTS/CTS exchange impedes the performance of the RTS/CTS scheme. Therefore, it is imperative to improve packet loss rate in WUSN to further improve data receiving rate. In the future work, other TDMA based MAC protocols will be evaluated and compared to the CDMA based MAC Protocol.

\section{ACKNOWLEDGEMENTS}

This work is supported in part by the National Science Foundation CAREER Award CNS-0953900.

\section{REFERENCES}

[1] I. F. Akyildiz and E. P. Stuntebeck, "Wireless underground sensor networks: Research challenges," Ad Hoc Networks Journal (Elsevier), vol. 4, pp. 669-686, July 2006.

[2] I. Demirkol, C. Ersoy, and F. Alagöz, "MAC protocols for wireless sensor networks: A survey," IEEE Communications Magazine, vol. 44, no. 4, pp. 115-121, 2006.

[3] X. Dong and M. C. Vuran, "A channel model for wireless underground sensor networks using lateral waves," in Proc. of IEEE Globecom '11, Houston, TA, December 2011.

[4] — "Return loss analysis of antennas in wireless underground sensor networks," submitted.

[5] K. Kredo and P. Mohapatra, "Medium access control in wireless sensor networks," Computer Networks (Elsevier), vol. 51, no. 4, pp. 961-994, March 2007.

[6] J. Polastre, J. Hill, and D. Culler, "Versatile low power media access for wireless sensor networks," in SenSys '04: Proceedings of the 2nd international conference on Embedded networked sensor systems, Baltimore, MD, USA, 2004, pp. 95-107.

[7] A. R. Silva and M. C. Vuran, "Communication with aboveground devices in wireless underground sensor networks: An empirical study," in Proc. of IEEE ICC'10, Cape Town, South Africa, May 2010, pp. 1-6.

[8] — , "Development of a Testbed for Wireless Underground Sensor Networks," EURASIP Journal on Wireless Communications and Networking, vol. 2010, 2010.

[9] _ - "Empirical evaluation of wireless underground-to-underground communication in wireless underground sensor networks," in Proc. of IEEE International Conference on Distributed Computing in Sensor Systems (DCOSS '09), Marina del Rey, CA, June 2009, pp. 231-244.

[10] Z. Sun and I. Akyildiz, "Connectivity in wireless underground sensor networks," in Proc. of IEEE Communications Society Conference on Sensor Mesh and Ad Hoc Communications and Networks (SECON '10), Boston, MA, 2010.

[11] M. J. Tiusanen, "Wireless Soil Scout prototype radio signal reception compared to the attenuation model," Precision Agriculture, vol. 10, no. 5, pp. 372-381, November 2008.

[12] M. C. Vuran and I. F. Akyildiz, "Channel model and analysis for wireless underground sensor networks in soil medium," Physical Communication, vol. 3, no. 4, pp. 245-254, December 2010.

[13] G. Zhou, T. He, S. Krishnamurthy, and J. A. Stankovic, "Models and solutions for radio irregularity in wireless sensor networks," ACM Trans. Sen. Netw., vol. 2, no. 2, pp. 221-262, 2006

[14] M. Zuniga and B. Krishnamachari, "Analyzing the transitional region in low power wireless links," in Proc. IEEE SECON '04, Santa Clara, CA, October 2004 